\title{
How do we survey IBD in Japan: chromoendoscopy versus white light?
}

\section{(ㄷ)(1) $(9$}

\author{
Authors \\ Yoshitaka Ueno $^{1,2}$, Shinji Tanaka ${ }^{1}$ \\ Institutions \\ 1 Department of Endoscopy, Hiroshima University \\ Hospital, Hiroshima, Japan \\ 2 Health Management and Promotion Center, Hiroshima \\ Atomic Bomb Casualty Council, Hiroshima, Japan
}

\author{
Bibliography \\ DOI https://doi.org/10.1055/a-1190-4039 | \\ Endoscopy International Open 2020; 08: E1018-E1020 \\ (c) Georg Thieme Verlag KG Stuttgart · New York \\ eISSN 2196-9736 \\ Corresponding author \\ Yoshitaka Ueno, Department of Endoscopy, Hiroshima \\ University Hospital, 1-2-3 Kasumi, Minami-ku, Hiroshima \\ 734-8551, Japan \\ Fax: +81-82-257-5194 \\ yueno@hiroshima-u.ac.jp
}

Patients with ulcerative colitis (UC) or Crohn's colitis have an increased risk of colorectal cancer compared with the general population. Because colitis-associated dysplasia is often subtle, flat or not endoscopically identifiable, the first surveillance guidelines recommended sampling four random biopsies from every $10 \mathrm{~cm}$ of colon in addition performing targeted biopsies of lesions suspected of containing dysplasia [1].

In earlier endoscopic eras, most dysplasia was "invisible" and discovered on random biopsy samples, but today, most dysplasia detected at surveillance colonoscopy is visible. Recent progress in endoscopic technologies, such as high-definition video endoscopy, and use of image enhancement methods such as chromoendoscopy, seem to explain the increased proportion of visible dysplastic lesions identified at surveillance colonoscopy [2].

In this issue of Endoscopy International Open, Ricardo et al report on a systemic review and meta-analysis of randomized controlled trials (RCTs) comparing various surveillance techniques [3]. The authors reviewed 17 RCTs totaling 2,457 patients. The evaluated outcomes were number of patients diagnosed with one or more dysplastic lesions, total number of dysplastic lesions detected, number of dysplastic lesions detected by directed biopsies, and procedure time. The authors found that dye-spraying chromoendoscopy detected more patients and dysplastic lesions than standard-definition white-light endoscopy (WLE). Although no difference was observed between dye-spraying chromoendoscopy and high-definition WLE or narrow-band imaging, the main outcomes favored numerically dye-spraying chromoendoscopy, except procedure time.
Chromoendoscopy, also known as dye-based image enhancement, using a dye solution of either methylene blue or indigo carmine applied onto the colonic mucosa to enhance contrast during surveillance colonoscopy, is generally performed either in a pancolonic fashion to detect lesions or a targeted fashion to allow for detailed viewing of an identified lesion. These agents are applied to the entire colonic mucosa with a spray catheter or the water jet channel of a standard colonoscope. The Surveillance for Colorectal Endoscopic Neoplasia Detection and Management in inflammatory Bowel Disease Patients: International Consensus Recommendations (SCENICICS) consensus reinforces the placement of panchromoendoscopy as the preferred technique for surveillance of dysplasia in inflammatory bowel disease (IBD) [4]. Accordingly, many Western scientific societies, such as the British Society of Gastroenterology and European Crohn's and Colitis organization, recommend use of panchromoendoscopy with targeted biopsies for IBD-colorectal cancer surveillance.

Despite these results and recommendations, panchromoendoscopy is not routinely used for IBD-colorectal cancer surveillance. This protocol carries additional costs for the equipment needed for dye spraying, and it is time consuming and requires an endoscopist who is familiar with the technique. Furthermore, some patients are not considered as candidates for chromoendoscopy because of inadequate bowel preparation or significant visible inflammation. Presence of active mucosal inflammation or post-inflammatory polyps may affect the images and procedures of chromoendoscopy. Mucosal healing may improve identification of dysplasia. Bowel preparation should be excellent to allow for detailed mucosal evaluation. 
Barriers to adoption of chromoendoscopy also include patient adherence. Historic data on patient adherence to colitis surveillance programs suggest that about half of patients drop out, negating the efficacy of surveillance.

Most studies demonstrating the superiority of chromoendoscopy over WLE with standard-definition colonoscopies had been done before the studies evaluating characterization of morphological features of dysplasia detected by high-definition WLE. Recently, several reports have been published about accurate endoscopic characterization of dysplasia. Sugimoto et al classified for the first time the morphological features of highgrade dysplasia using the SCENIC consensus [5]. The authors detected all lesions by targeted biopsy sampling without panchromoendoscopy and found that high-grade dysplasia is frequently associated with a flat/superficial elevated area and red discoloration. The authors performed regular white-light colonoscopy, followed by $0.2 \%$ to $0.4 \%$ indigo-carmine dye spraying after suspected lesions were recognized. lacucci et al have developed, validated, and reproduced a new endoscopic classification (FACILE; Frankfurt Advanced Chromoendoscopic Ibd LEsions) using all imaging modalities for diagnosis of dysplasia in IBD [6]. Flat shape, irregular surface, vascular pattern, and signs of inflammation predicted dysplasia.

A recent meta-analysis of 10 studies (494 patients) compared dye-based chromoendoscopy with standard-definition WLE and high-definition WLE [7]. RCTs in the literature showed a small benefit of chromoendoscopy over standard-definition WLE, but not over high-definition WLE. New and advanced endoscopic technologies, such as increased resolution of highdefinition endoscope, significantly improved resolution of the images compared to conventional WLE, as dysplasia became easier to see from the greater detail of the images [8]. When assessing the comparative network analysis, we must keep in mind that inclusion of studies published in the first decade of the 2000 s could be biased by use of the previous generation of endoscopes with standard WLE.

In Japan, there is no concrete consensus on management of colitis-associated neoplasia. According to the discussion on the basis of a questionnaire survey completed by nine Japanese expert panelists from high-volume centers for patients with UC, WLE used high definition as the main observation method of surveillance colonoscopy [9]. Recently Watanabe et al reported that results of a trial of patients with UC who were randomized to surveillance colonoscopy showed that targeted biopsy is as effective as a random biopsy for detection of neoplasia in UC [10]. This study was performed in 52 Japanese institutions. As discussed in the literature, panchromoendoscopy is not generally used in surveillance colonoscopy in Japan. Chromoendoscopy is generally performed in a targeted fashion to allow for detailed viewing of an identified lesion. In the authors' institution, high-magnification colonoscopy with indigo carmine chromoendoscopy is routinely performed after they suspect presence of dysplasia in patients with IBD. Morphologically, dysplasia in IBD appears to be slightly elevated, completely flat, or slightly depressed as compared with the surrounding mucosa. To detect it, the authors look for presence of a slightly elevated lesion, focal friability, obscure vascular pattern, discoloration (uneven redness or a patch or redness), villous mucosa (velvety appearance), and irregular nodularity. Examination with varying expansion of the colon (after increasing and decreasing air insufflation) also improves visualization of the subtle-appearing flat and depressed neoplasms. Once a suspicious lesion is identified, the mucosal surface is washed with a detergent to remove mucus and bubbles before detailed observation. Then approximately $0.4 \%$ of indigo carmine dye is sprayed directly from a $60-\mathrm{mL}$ syringe through the biopsy channel. In our experience, we realized that some dysplasia found by chromoendoscopy could be identified with high-definition WLE by careful observation of subtle color differences or mild friability. Chromoendoscopy is used for characterization of the detected lesions found by WLE.

A novel endoscopic system and colonoscope with a dual-focus function has recently become available in Western countries. This enables dual-focus near-field magnification by pushing a single button to closely examine the surface structure and capillary network of the mucosa. Nishiyama et al reported that chromoendoscopy with a magnifying endoscope is useful for differentiating between neoplastic and non-neoplastic lesions by assessing the high residual density of pits and irregular pit margins under magnification [11].

Although we still do not have the perfect method, techniques for defining the mucosa in detail during endoscopy are rapidly evolving with various new endoscopic systems. The search for a gold standard method for colon cancer surveillance in patients with IBD continues with the goal of identifying one that can be widely used.

\section{Competing interests}

The authors declare that they have no conflict of interest.

\section{References}

[1] Winawer S, Fletcher R, Rex D et al. Colorectal cancer screening and surveillance: clinical guidelines and rationale-update based on new evidence. Gastroenterology 2003; 124: 544-560

[2] Beaugerie L, Itzkowitz SH. Cancers complicating inflammatory bowel disease. N Engl J Med 2015; 372: 1441-1452

[3] Ricardo R, Igor R, Diogo M et al. Surveillance in inflammatory bowel disease: is chromoendoscopy the only way to go? A systematic review and meta-analysis of randomized clinical trials Endosc Int Open 2020; 08: 578-590

[4] Laine L, Kaltenbach T, Barkun A et al. SCENIC international consensus statement on surveillance and management of dysplasia in inflammatory bowel disease. Gastrointest Endosc 2015; 81: 489-501

[5] Sugimoto S, Naganuma M, Iwao Y et al. Endoscopic morphologic features of ulcerative colitis-associated dysplasia classified according to the SCENIC consensus statement. Gastrointest Endosc 2017; 85: 639-646

[6] lacucci M, McQuaid K, Gui XS et al. A multimodal (FACILE) classification for optical diagnosis of inflammatory bowel disease associated neoplasia. Endoscopy 2019; 51: 133-141

[7] Feuerstein JD, Rakowsky S, Sattler L et al. Meta-analysis of dye-based chromoendoscopy compared with standard-and high-definition 
white-light endoscopy in patients with inflammatory bowel disease at increased risk of colon cancer Gastrointest Endosc 2019; 90: 186-195

[8] lacucci M, Kaplan GG, Panaccione R et al. A randomized trial comparing high definition colonoscopy alone with high definition dye spraying and electronic virtual chromoendoscopy for detection of colonic neoplastic lesions during IBD surveillance colonoscopy. Am J Gastroenterol 2018; 113: 225-234
[9] Oka S, Uraoka T, Watanabe $\mathrm{K}$ et al. Endoscopic diagnosis and treatment of ulcerative colitis-associated neoplasia. Dig Endosc 2019; 31: 26-30

[10] Watanabe T, Ajioka Y, Mitsuyama K et al. Comparison of targeted vs random biopsies for surveillance of ulcerative colitis-associated colorectal cancer. Gastroenterology 2016; 151: 1122-1130

[11] Nishiyama S, Oka S, Tanaka $S$ et al. Is It possible to discriminate between neoplastic and nonneoplastic lesions in ulcerative colitis by magnifying colonoscopy? Inflamm Bowel Dis 2014; 20: 508-513 